(GlideScope ${ }^{\circledR}$ ) in the management of a difficult airway. Can J Anesth 2003; 50: 611-3.

\section{Warming the tracheal tube and kinking}

To the Editor:

We read with interest the letter on intratracheal kinking of an endotracheal tube by Lee et al. ${ }^{1}$ The authors highlighted that, in addition to various known causes of obstruction of the endotracheal tube, intratracheal kinking at the site where the inflating lumen opens into the cuff can also cause its obstruction. Their observation is similar to that of Singh et al. ${ }^{2}$ where the authors could barely pass the endotracheal tube through the nasal cavity following soaking it in warm water. It is well known that thermal softening of the polyvinyl chloride tracheal tube can lead to its distortion and obstruction. ${ }^{3}$ In the case reported by Lee et al., we postulate that warming the tube to soften it contributed to kinking at the tube's weakest point, i.e., the site where the inflating lumen opens into the cuff.

We feel that the practice of softening the endotracheal tube should be avoided. If one is unable to intubate with a particular size tube it is better to use a smaller size that can be inserted without causing trauma.

Anil Agarwal MD

Lucknow, India

\section{References}

1 Lee YW, Lee TS, Chan KC, Sun WZ, Lu CW. Intratracheal kinking of endotracheal tube. Can J Anesth 2003; 50: 311-2.

2 Singh B, Gombar KK, Chhabra B. Tracheal tube kinking (Letter). Can J Anaesth 1993; 40: 682.

3 Alaya JL, Coe A. Thermal softening of tracheal tubes: an unprecedented hazard of the Bair Hugger active patient warming system. Br J Anesth 1997; 79: 543-5.

\section{Nasotracheal intubation, epistaxis and atelectasis in a patient with anhidrotic ectodermal dysplasia}

To the Editor:

Anhidrotic ectodermal dysplasia (AED) is a rare hereditary disorder affecting ectodermally-derived tissues and organs. It is characterized by hypohidrosis, hypodontia and hypotricosis. ${ }^{1}$

A five-year-old boy $(26 \mathrm{~kg}, 116 \mathrm{~cm})$ with AED was scheduled for extraction of impacted teeth under general anesthesia. A nasotracheal tube (inner diameter, 5.0 $\mathrm{mm}$ ) was softened with warm saline before intubation. Anesthesia was induced with $5 \%$ sevoflurane in combination with nitrous oxide and oxygen via a face mask. After venous cannulation, $3 \mathrm{mg}$ vecuronium bromide was used to facilitate tracheal intubation. The tube was inserted into the right naris. Resistance was not felt during transit of the tube through the nasal passageway. However, blood was found in the pharynx and hindered intubation under direct laryngoscopic visualization. Aspiration resulted in the immediate removal of a considerable quantity of blood. As we could visualize the vocal cord with a laryngoscope, the tube was placed in the trachea with the aid of a Magill forceps. A decrease in $\mathrm{SpO}_{2}$ was noted following the induction of epistaxis by the nasotracheal intubation with diminished respiratory sounds being evident in the right upper lung field. A chest $x$-ray indicated atelectasis and an obstructing clot was removed by bronchoscopy from the right upper lobe bronchus. This resulted in an improvement in $\mathrm{SpO}_{2}$. In the ward three hours after extubation, the atelectasis was no longer evident on a chest $x$-ray. On the first postoperative day, hematological examination revealed a mild inflammatory state and the patient was treated with antibiotics. There was no evidence of a respiratory tract infection and the exact cause of the inflammatory state was not determined. He was discharged on the fifth postoperative day.

Patients with AED are predisposed to epistaxis because of poor humidification of inspired air leading to generalized drying and crusting of the airway. ${ }^{2}$ To our knowledge, our case is the first report of epistaxis and atelectasis following nasotracheal intubation in a patient with AED. We believe that imperfect suctioning of blood in the pharynx resulted in blood entering the bronchus when tracheal intubation was performed. The alpha-adrenergic agonist oxymetazoline is effective for the prevention of epistaxis associated with nasotracheal intubation ${ }^{3}$ and may, therefore, be useful in a patient with AED.

In conclusion, specially in patients with AED, it is necessary to perform nasotracheal intubation very delicately in order to prevent epistaxis and associated complications.
Hideaki Ishii MD
Ippei Watanabe MD
Konosuke Watanabe MD
Chie Kobayashi MD
Masanori Maruyama MD
Hiroshi Baba MD
Niigata, Japan 